\title{
WLAN Cell Handoff Latency Abatement Using an FPGA Fuzzy Logic Algorithm Implementation
}

\author{
Roberto Sepúlveda, Oscar Montiel-Ross, Jorge Quiñones-Rivera, and Ernesto E. Quiroz
}

Instituto Politécnico Nacional-CITEDI, Avenida del Parque No. 1310, 22510 Tijuana, BC, Mexico

Correspondence should be addressed to Roberto Sepúlveda, r.sepulveda@ieee.org

Received 5 May 2012; Accepted 29 May 2012

Academic Editor: Oscar Castillo

Copyright ( $) 2012$ Roberto Sepúlveda et al. This is an open access article distributed under the Creative Commons Attribution License, which permits unrestricted use, distribution, and reproduction in any medium, provided the original work is properly cited.

Following the path toward $4 \mathrm{G}$ set by its wireless siblings LTE and WiMax, IEEE 802.11 technology, universally known as WiFi, is evolving to become a high data rate QoS-enabled mobile platform. The IEEE $802.11 \mathrm{n}$ standard yields data rates up to 450 Mbp s and the 802.11e standard ensures proficient QoS for real-time applications. Still in need of better performance, multicell environments that provide extended coverage allow the mobile station nomadic passage beyond a single cell by means of cell dissociation-association process known as handoff. This process poses a challenge for real-time applications like voice over IP ( $150 \mathrm{~ms}$ maximum delay) and video (200-400 ms) sessions, to give the user a seamless cell-crossing without data loss or session breakage. It presented an approach of a predictive fuzzy Logic controller to reduce the channel scanning process to a tenth of the standard time, and its efficient FPGA implementation to speed up the processing time. The algorithm of the fuzzy controller was implemented in C language. Experimental results are provided.

\section{Introduction}

WiFi (Wireless Fidelity) wireless connectivity keeps permeating virtually in all categories of consumer electronics devices. Besides the traditional application in laptops, tablets, and dual-mode (cellular-WiFi) handsets, WiFi connectivity is lurking into TVs, media players, gaming consoles, connected home, and so forth. Production of WiFi devices reached nearly 1.1 billion in 2011 and is expected to double by 2015. The growth is a solid $25-39 \%$ in health, fitness, medical applications, smart meters, and automation products; a staggering $109 \%$ increase was predicted in automotive applications such as infotainment systems, navigation, and traffic monitoring [1].

WiFi relies on the IEEE 802.11 [2] radio standards, the WiFi Protected Access (WPA) and WPA2 security standards, and the EAP (Extensible Authentication Protocol) authentication standard. A WiFi Access Point (AP) provides wireless connectivity to Mobile Stations (MS) within its cell coverage (30-110 yards), varying by commercial implementation. A multi-cell ensemble can be fitted to a particular setting to cover a wider area with multiple APs (Figure 1). MS handoff $(\mathrm{HO})$ from one cell to another provides seamless connectivity to itinerant users moving beyond the radio range of its currently associated AP to enter a neighboring AP's basic service set (coverage area). During the handoff process, management frames are exchanged between the MS and the AP. Also the APs involved exchange context information pertaining to the MS. Table 1 presents the standards that represent the generations in the evolution of WiFi [3].

Voice was not originally supported by WiFi, but the convergence trend brought about by the IP protocol thrusts voice over IP (VoIP) into the digital multimedia stream. Also, the great advancements in VoIP compression technology and the deployment of carrier-class VoIP networks have made possible extremely low prices. Adhering to the VoIP drive, IEEE $802.11 \mathrm{n}$ incorporates quality of service (QoS) mechanisms on a per cell basis, with stringent performance requirements, that is: (a) packet loss of less than $1 \%$ with no burst losses; (b) latency of less than $50 \mathrm{~ms}$; (c) maximum jitter of less than $50 \mathrm{~ms}$; (d) VoIP precedence over any other data stream being handled by the AP. Nevertheless, $\mathrm{HO}$ is not among the high priority issues. An inefficiently handoff process might cause call interruption or at best, uncomfortable loss of information. Situations that can push 


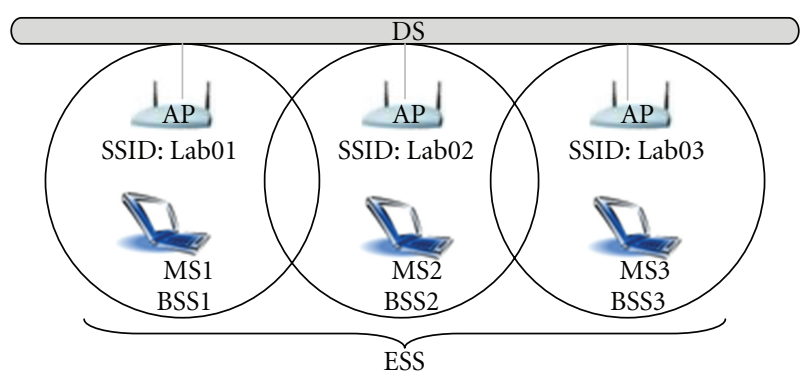

DS: Distribution system AP: Access point SSID: Service set identifier MS: Mobile station BSS: Basic service set ESS: Extended service set

FIGURE 1: Extended and basic service sets coverage.

TABLE 1: Wi-Fi generations.

\begin{tabular}{lcc}
\hline Wi-Fi technology & Frequency band & Maximum data rate \\
\hline $802.11 \mathrm{a}$ & $5 \mathrm{GHz}$ & $54 \mathrm{Mbps}$ \\
$802.11 \mathrm{~b}$ & $2.4 \mathrm{GHz}$ & $11 \mathrm{Mbps}$ \\
$802.11 \mathrm{~g}$ & $2.4 \mathrm{GHz}$ & $54 \mathrm{Mbps}$ \\
& $2.4 \mathrm{GHz}, 5 \mathrm{GHz}, 2.4$ or & \\
$802.11 \mathrm{n}$ & $5 \mathrm{GHz}($ selectable), or 2.4 & $450 \mathrm{Mbps}$ \\
& and $5 \mathrm{GHz}$ (concurrent) & \\
\hline
\end{tabular}

the HO process to its limits are: (a) WLAN cells have a small coverage area, and a fast moving MS may produce frequent, short-time interval handoffs. (b) There is a latency $(\approx 300 \mathrm{msec}$.) [4] involved in the handoff process during which the MS is unable to send or receive any kind of traffic. (c) VoIP requires one-way end-to-end delay of less than $150 \mathrm{~ms}$ [5].

Faster processing time during $\mathrm{HO}$ is desirable. The continuous breakthroughs in Very Large Scale Integrated Circuits (VLSI), mainly in Field-Programmable Gate Array (FPGA) and the Applied Specific Integrated Circuit (ASIC) domains, and also in the development of new programming tools that allow to undertake complex digital designs to creation in a very small time affords to design and implement high-performance systems embedded into an integrated circuit. Other features about FPGA are that they consume low power and can be reprogrammable in field; hence, there is an increasing interest in using FPGA devices to design digital controllers, and a growing interest in control systems that require a real time operation based on fuzzy logic. Nowadays, the studies and proposals to implement fuzzy systems into an FPGA keep adding [6-8], including interesting FPGA implementations of type 1 fuzzy inference systems (FIS) in electrical vehicles, such as [9]. Some proposals implement type 2 FIS [10-12], as well as others that focus on the software development for coding a highspeed defuzzification stage for a type 2 FIS [13]. More recent works include [14], where an interval type 2 FIS KarnikMendel-type reducer is designed, tested, and implemented.

In order for QoS delay constraints for VoIP to be fulfilled during an intercell handover, we implement a fuzzy logic fast handoff algorithm in an FPGA, which instead of scanning multiple channels predicts the best fitted channel and scans only the selected channel, reducing the lengthier part of the HO process up to an eleventh, since eleven is the maximum number of adjacent cell channels the standard allows. The following sections of the paper are organized as follows. Section 2 explains 802.11 handoff procedure, a selection of other variants looking to reduce the time consumed in the process, and our solution. Section 3 discusses the FPGA implementation. Section 4 presents the simulation results and Section 5 concludes the paper.

\section{Intercell Handoff Procedures}

2.1. 802.11 Handoff Standard. The main requirements an HO procedure must fulfill are low latency, scalability, minimum drop-off and fast recovery, QoS (maintained or renegotiated), and security.

When an MS approaches the limits of its BSS, the intensity and quality of its signal deteriorate, approaching a link breaking point. The AP's radio signals are severely affected by various environmental factors, such as distancerelated attenuation, obstacle-induced fading, multipath signal aggregation, and other radiofrequency sources interference. As the MS's Received Signal Strength (RSS) from the source AP diminishes, the MS receives strong signals from neighboring APs, so that when the local signal faints to a predefined threshold (i.e., $80 \mathrm{dBm}$ ), the $\mathrm{HO}$ process is initiated [15]; see Figure 2.

IEEE 802.11 contemplates two channel configurations to carry on the handoff procedure: single-channel roaming and multichannel roaming. In this paper we will be dealing with the latter, since the channel-scanning process takes longer than the former.

2.1.1. Multichannel Roaming. Each AP is assigned a beacon channel, to transmit a reference signal at $100 \mathrm{~ms}$ intervals [16]. Up to eleven channels can be used in an Extended Service Set (ESS) simultaneously (limit stated in 802.11 standard) [17]. A polling scheme is used by the MS to scan each beacon-frame channel carrier generated by all surrounding APs. When the signal quality falls below the "start cell search" threshold, the MS sends a probe by each channel, requesting an immediate transmission of a beacon signal (Figure 3). Neighboring APs send a response frame in a sequential fashion, so the MS can perform a real time measure of the received power from all APs. When an AP's signal is better than the threshold, the MS initiates the transference to the selected AP's neighboring cell. The target AP authenticates, associates with the MS, and allocates resources. Following communicates with the source AP via an inter-accesspoint protocol [17]. Finally, the source AP disassociates from the MS. The time elapsed in the HO execution is variable, depending on the number of channels (cell ensemble) present for scanning. When the eleven channel are used, the time consumed is around $300 \mathrm{~ms}$ [4].

2.2. Related Work. Mishra et al. [18] analyze handoff latency at the link layer, demonstrating that multichannel-scanning 


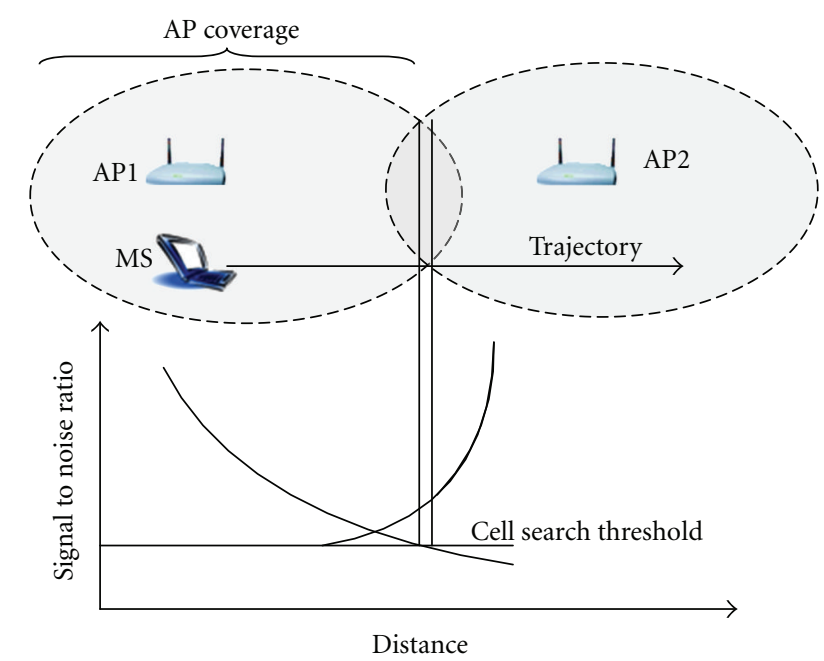

FIGURE 2: Intercell overlap zone for handoff realization.

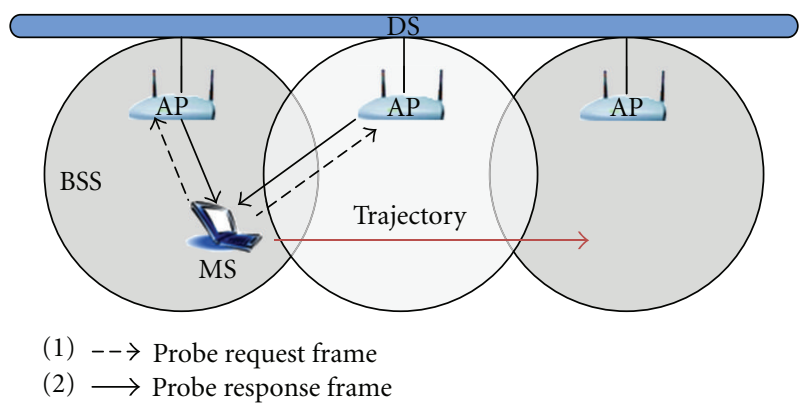

FIgURE 3: Scan sweep.

contributes with most of the total handoff delay, yielding poor QoS for time-sensitive applications. Shin et al. [19] use a scanning algorithm to select a subset of channels and build a channel mask. This mask is used in the next handoff to scan only the predefined subset reducing the scanning delay by $30 \%$ to $60 \%$ compared to the standard procedure. Li et al. [20] use a neighbors' graphics caching mechanism. The mobile station will know beforehand the channels used by the neighboring access points, so there is no need to explore all available channels, reducing scanning latency. Chang et al. [21] assess the average change in the MS's RSS, pinpointing the more appropriate AP to serve the handoff. Song et al. [22] introduce improvements to the mobile IPv6 fast handover protocol in a subnet to subnet MS transference. Purushothaman and Roy's work [4] reduces the number of channels to scan by using a client-based database which stores information about the APs' channel numbers with higher RSS. Ong and Khan [23] eliminate the channel sweep stage based on the traditional power signal metrics. The HO triggering reference is the number of VoIP packets lost, which should not exceed $2 \%$ of the total sent in a period of time. This approach cuts $90 \%$ of the 802.11 standard handoff time [4]. Nevertheless, the downside is the cost of greater network complexity brought about by the need of an increased network-wide information exchange.
Some works have relied on the use of fuzzy logic (FL) in diverse aspects of the WLAN operation. Patil and Kolte [24] describe a five-parameter FL algorithm for handoff optimization. Nevertheless, it is generic and does not apply to the 802.11 WLAN case. Gharehbaghi and Badamchizadeh [25] perform a comparison of four FL algorithms for congestion control, a special high volume packet arrival situation of the target AP which causes a service denial to the approaching MS.

Our proposal relies on the link layer received-signalpower detection already in use, and the calculation of the direction of the MS in relation to the neighboring APs (used in [20]), to build a two-input Predictive Fuzzy Logic Control (PFLC) designed to compute in advance in which AP has the highest aptitude value to admit the MS when handoff initiation becomes necessary. So, in order to conform to the IEEE.802.11 triggering process, only one channel is scanned. Our proposal, thus, approaches the time reduction obtained by [23], but modifications are confined to the MS, instead of the whole network.

\section{Cell Handoff Fuzzy Logic Controller Design and Implementation}

3.1. Predictive Fuzzy Logic Controller. Figure 4 illustrates the Predictive Fuzzy Logic Controller (PFLC) participation in the HO process. At $100 \mathrm{msec}$, time intervals the APs broadcast beacon signals. The MS receives the RSS information from each channel and the PFLC performs an analysis that yields the channel number identifying the AP with the highest aptitude value at that time. In this manner, the MS has an updated knowledge of the best fitted AP to associate with. So, when the AP1 signal quality falls to the search threshold, triggering the handoff, the MS knows, among the eleven contiguous AP's channels, that AP2 has the higher aptitude. The standard's scanning operation is performed only once on the preselected channel.

A Mamdani-type fuzzy system was designed to predict, from a group of cells, which AP is best fitted to serve the itinerant MS. Figure 4 illustrates the two-input-oneoutput fuzzy controller; the same fuzzy system can be seen with more details in Figure 11. The first entry represents the Average Signal Intensity (ASI), calculated at two-second intervals from the beacon signal received by the mobile station every $100 \mathrm{msec}$. ASI constitutes a major metrics typically used in wireless systems to measure signal quality for purposes of performing the handoff. While the MS beacon signal does not fall to cell search threshold (set to $80 \mathrm{dBm}$ for this work), the handoff process is not triggered. The second input is the Signal Intensity Variation (SIV) parameter that provides information on the direction of the MS with respect to the AP (approaching or distancing).

Both inputs feed the fuzzy controller, which based on historic data and knowledge rules allow it to estimate the aptitude value of that particular AP. The same calculation is performed for al AP channels, and the highest aptitude output indicates which will be the target AP. 


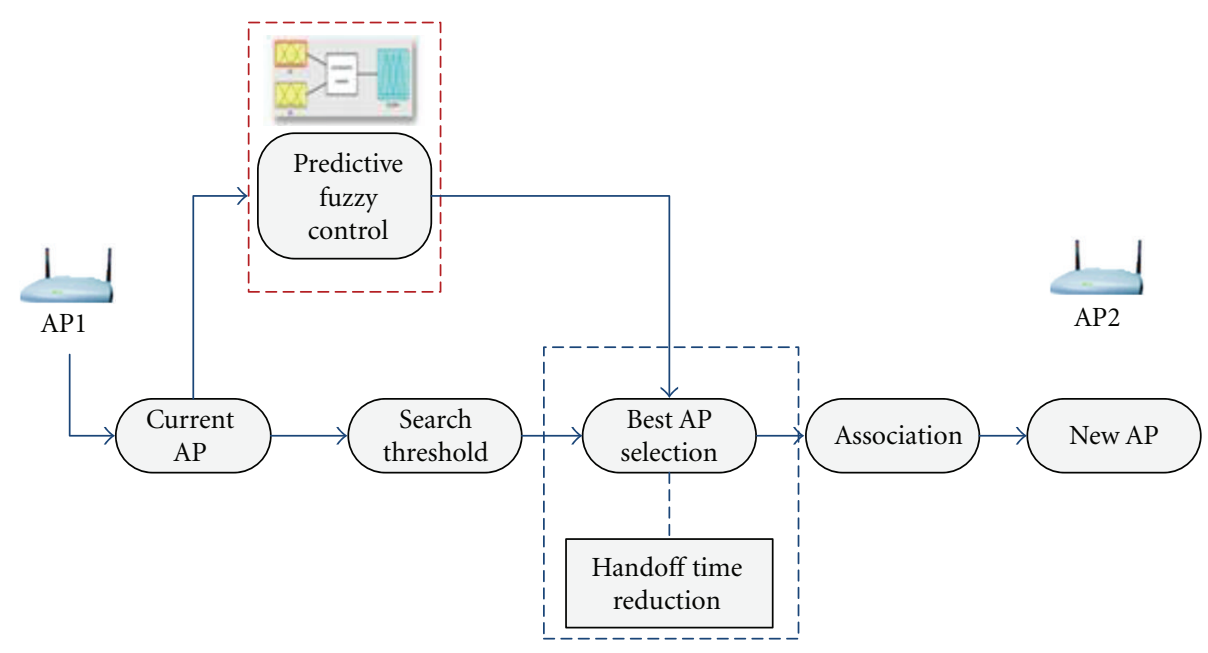

FIgURE 4: Predictive handoff scheme.

The inference mechanism applied is "max-min," widely known as the Mamdani method, characterized by its implementation simplicity and effectiveness. Also, the centroid method is used, because it provides more representative results from the output-weighted values of various membership functions.

The methodology to define membership functions for the three variables ranges (two inputs, one output) is described as follows.

(i) Input Variable: Average Signal Intensity. Four membership functions were set forth for ASI: low, medium, good, and excellent. The first and last are trapezoidal and the rest triangular, as shown in Figure 5.

The choice of the linguistic terms correspond to the quality of received signal.

The expression proposed by Chang et al. [21] is used to obtain the ASI received by the mobile station.

$$
\operatorname{ASI}(t)=\frac{\sum_{t=1}^{n} \operatorname{SSbeacon}(t, i)}{n} .
$$

The universe of discourse proposed for the ASI variable is shown in Figure 5, ASI is expressed by (1), where $t$ is the current time, $n$ represents the number of beacon frames received at time $t$, and finally $\operatorname{SSbeacon}(t, i)$ represents the intensity of the signal received at time " $t-i$ " (Figure 6).

(ii) Input Variable: Signal Intensity Variation (SIV). For the relative position of an MS with respect to a specific AP, SIV represents the rate of change of two ASI readings made two seconds apart. Three membership functions were defined for SIV, whose linguistic terms are "negative," "zero", and "positive," being two of trapezoidal shape, and one triangular as illustrated in Figure 7.

The expression to calculate SIV is given in (2). $\mathrm{SSa}(t)$ is the ASI value at the present time, and SSa $(t-k)$ represents an

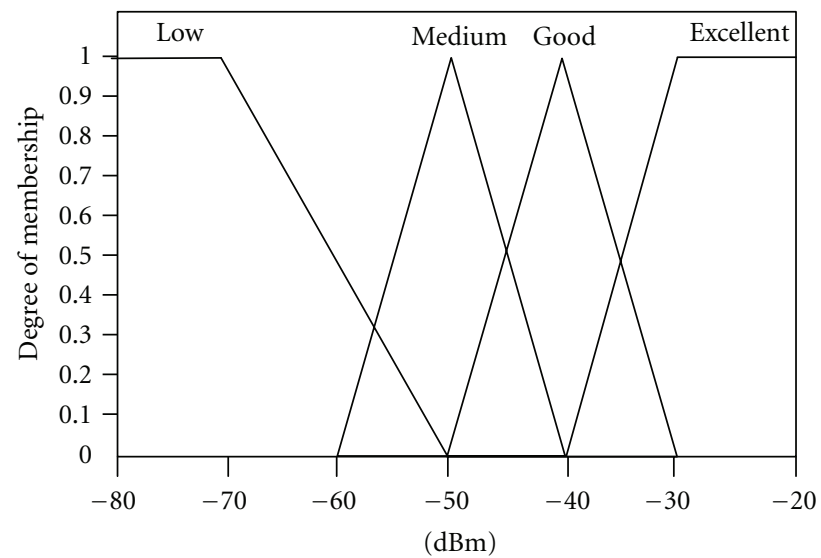

FIGURE 5: Average signal intensity in $\mathrm{dBm}$.

ASI reading $k=2$ seconds before. The range for this variable is set to $(-3,3)$

$$
\operatorname{SIV}(t)=\frac{\operatorname{SSa}(t)-\operatorname{SSa}(t-k)}{t-(t-k)} .
$$

Thus, the variation of signal intensity contains information about the speed of movement of the MS and about direction of the MS in reference to the AP (Figure 8). A positive value is indicative of displacement towards the AP and a negative value, of moving apart.

The traditional handoff scheme considers only the ASI, whereas ASI and SIV yield a better picture of the MS behavior.

(iii) Variable Output: Aptitude. Aptitude represents the decision taken on the basis of historic data of the two inputs, ASI and SIV. Five membership functions were considered appropriate for this variable: negative, small negative, zero, small positive, and positive, as shown in Figure 9. The range is $(-2,2)$. 


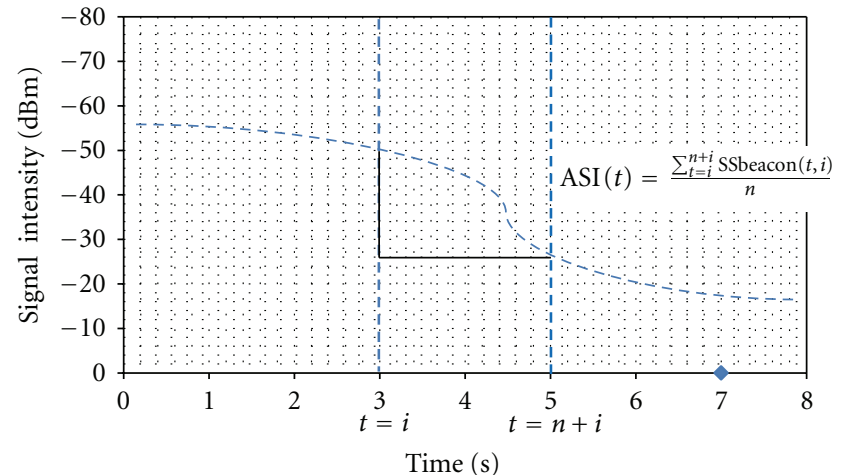

FIGURE 6: Interval time and the formula for the ASI calculation.

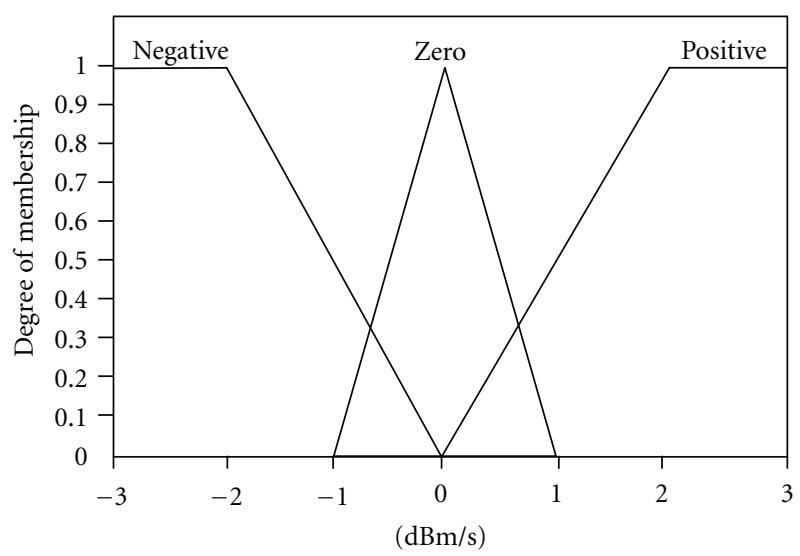

FIGURE 7: Signal intensity variation.

The fuzzy system's decision-making capability is defined by its knowledge base, comprised by 12 rules.

(1) If ASI is excellent and SIV is positive then aptitude is positive.

(2) If ASI is excellent and SIV is zero then aptitude is positive.

(3) If ASI is excellent and SIV is negative then aptitude is small positive.

(4) If ASI is good and SIV is positive then aptitude is positive.

(5) If ASI is good and SIV is zero then aptitude is small positive.

(6) If ASI is good and SIV is negative then aptitude is zero.

(7) If ASI is media and SIV is positive then aptitude is small positive.

(8) If ASI is medium and SIV is zero then aptitude is zero.

(9) If ASI is medium and SIV is negative then aptitude is small negative.

(10) If ASI is low and SIV is positive then aptitude is zero.

(11) If ASI is d Low own and SIV is zero then aptitude is small negative.

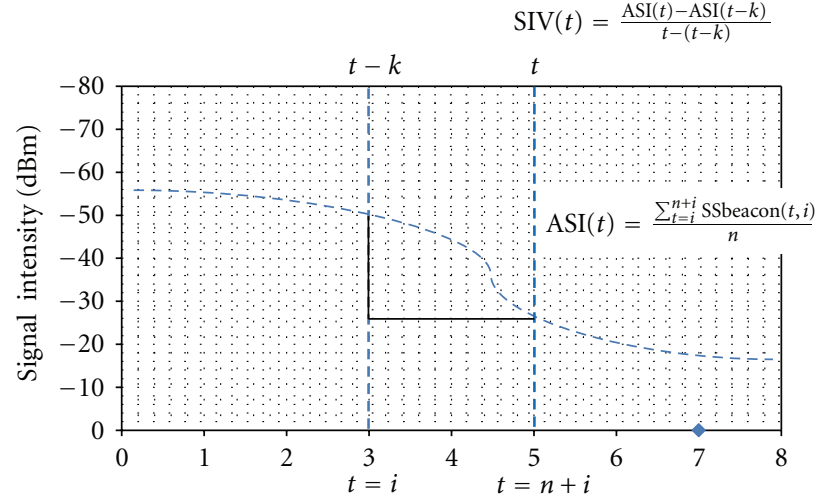

Time (s)

FiguRe 8: FLPC input parameters.

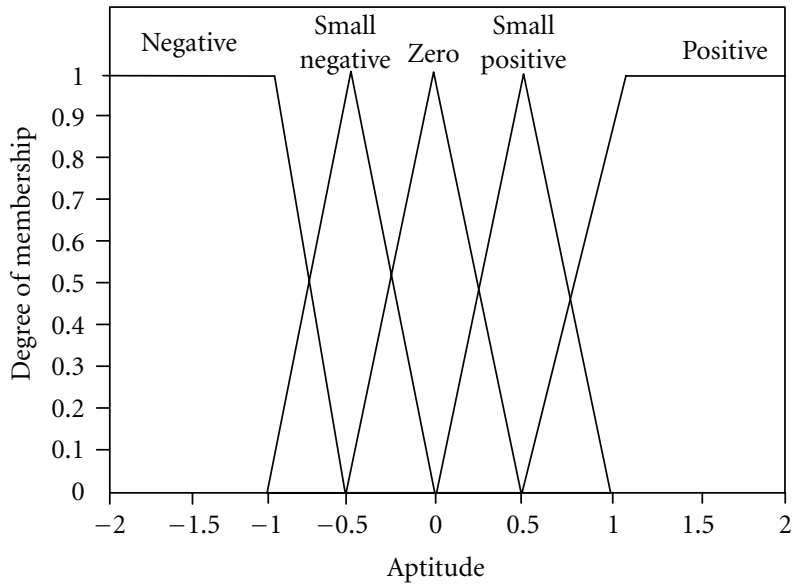

FIGURE 9: Output variable: aptitude value.

TABLE 2: Fuzzy system rules matrix.

\begin{tabular}{lccc}
\hline ASI & & SIV & \\
& Positive & Zero & Negative \\
\hline Excellent & Positive & Positive & Small positive \\
Good & Positive & Small positive & Zero \\
Medium & Small positive & Zero & Small negative \\
Low & Zero & Small negative & Negative \\
\hline
\end{tabular}

(12) If ASI is low and SIV is negative then aptitude is negative.

The number of linguistic terms of each entry sets the number of rules, in this case $4 \times 3=12$ rules. The knowledge base arrangement is illustrated by the matrix shown in Table 2. The decision taken by the predictive fuzzy control is clearly defined by the two inputs values. Table 3 provides a synthesis of the values that define the membership functions.

3.2. Fuzzy Logic Implementations. Hardware implementation was carried on using the "SmartFusion Evaluation" development kit Actel FPGA A2F200M3F (Figure 10), whose main specs are as follows: $200 \mathrm{~K}$ gates, $256 \mathrm{~Kb}$ memory 
TABle 3: Parameters of membership functions.

\begin{tabular}{|c|c|c|c|c|c|c|}
\hline Linguistic variable & Linguistic term & Type of function & \multicolumn{4}{|c|}{ Parameters } \\
\hline \multirow{4}{*}{ Average signal intensity } & Low & Trapezoidal & -80 & -80 & -70 & -50 \\
\hline & Medium & Triangular & -60 & -50 & -40 & \\
\hline & Good & Triangular & -50 & -40 & -30 & \\
\hline & Excellent & Trapezoidal & -40 & -30 & -20 & -20 \\
\hline \multirow{3}{*}{ Signal intensity variation } & Negative & Trapezoidal & -3 & -3 & -2 & 0 \\
\hline & Zero & Triangular & -1 & 0 & 1 & \\
\hline & Positive & Trapezoidal & 0 & 1 & 3 & 3 \\
\hline \multirow{5}{*}{ Aptitude } & Negative & Trapezoidal & -2 & -2 & -1 & -0.5 \\
\hline & Small Negative & Triangular & -1 & -0.5 & 0 & \\
\hline & Zero & Triangular & -0.5 & 0 & 0.5 & \\
\hline & Small Positive & Triangular & 0 & 0.5 & 1 & \\
\hline & Positive & Trapezoidal & 0.5 & 1 & 2 & 2 \\
\hline
\end{tabular}

flash, $64 \mathrm{~Kb}$ SRAM, $20 \mathrm{Mhz}$ crystal oscillator [26], and 32 bits ARM Cortex M3 soft processor (Figure 11). The FPGA using its logical blocks, allows customization through the "Libero-" integrated design software, firmware edition, and compilation in C language.

Fuzzytech software [27] was used to design the FIS. The PFLC was set to 16 bits resolution and was evaluated using the C-function EvalFis(float Var_ASI, float Var_SIV), which receives the input values ASI and SIV and returns the result of the output variable Aptitude. Figure 11 illustrates the embedded FLPC design. We used the C language tools of Fuzzytech to develop the algorithm of the PFLC.

\section{Experiments and Results}

This section presents some of the tests performed in order to validate the PFLC design and performance.

4.1. Itinerant MS in Source Cell: No Handoff. In its simplest expression, an MS moves around within the limits of its serving cell. As usual, beacon frames are received at $100 \mathrm{msec}$. intervals and used to calculate ASI. SIV is updated every 2 sec., as well as the aptitude value. Since a user can find obstacles and move around to avoid them, signal intensity by itself is not a good reference to determine an MS's direction. So, the SIV provides this information, as stated before in (2).

Table 4 shows some sample values of the AP's signal intensity received by the MS.

The first value is close to the minimum detectable signal strength, indicating that the MS is far from the AP and near a boundary. As the MS moves, the values start increasing, which implies that the MS is getting closer to the AP. Table 5 shows various ASI values obtained.

As illustrated in Figure 11, the PFLC performs its decisions based on the two inputs (ASI, SIV) and its knowledge base, yielding a numerical result, known as aptitude value. In our design, +3 is the maximum aptitude, and -3 the minimum. Figure 12 presents an instance of calculation.

4.2. Itinerant MS Crossing Cell Boundaries in an Eleven Aps Ensemble. The simulation considers an MS receiving the

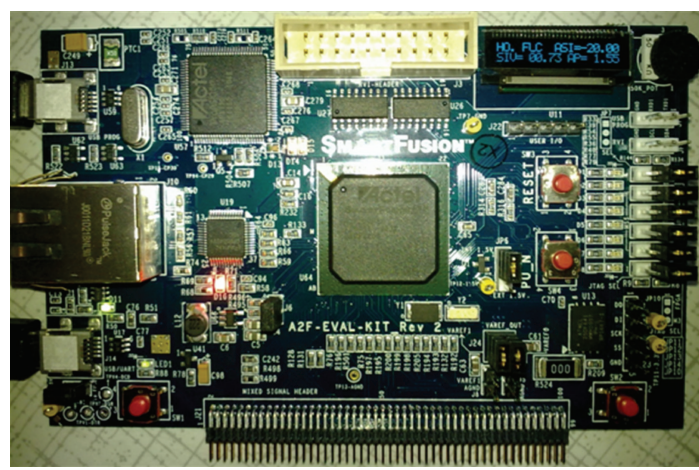

Figure 10: Actel development board for FPGA.

TABLE 4: Intensity values received by the MS.

\begin{tabular}{lcc}
\hline Signal intensity $(\mathrm{dBm})$ & Sequence of beacons & Time in $(\mathrm{msec})$. \\
\hline-79.12 & 1 & 100 \\
-78.73 & 2 & 200 \\
-78.52 & 3 & 300 \\
-77.92 & 4 & 400 \\
-77.40 & 5 & 500 \\
$\ldots$ & $\ldots$ & $\ldots$ \\
-78.00 & 20 & 2000 \\
\hline
\end{tabular}

beacon frames from a group of eleven surrounding cells. To illustrate cell crossings and related handoff, Figure 13 shows four cells only.

The MS's FLPC performs ASI-SIV calculations every two seconds to obtain the aptitude value for eleven APs and select the one with the higher value; in this way, the ARM processor acquires the values of the eleven ASI and SIV signals, then the embedded FLPC executes each inference and stores each APs output result, then selects the maximum value.

When the proper conditions are met to trigger a handoff, the best positioned AP and channel are known. As the MS travels along the trajectory, it navigates through different coverage areas, aptitude values are computed, and beacon signals from various cells become significant and competitive 


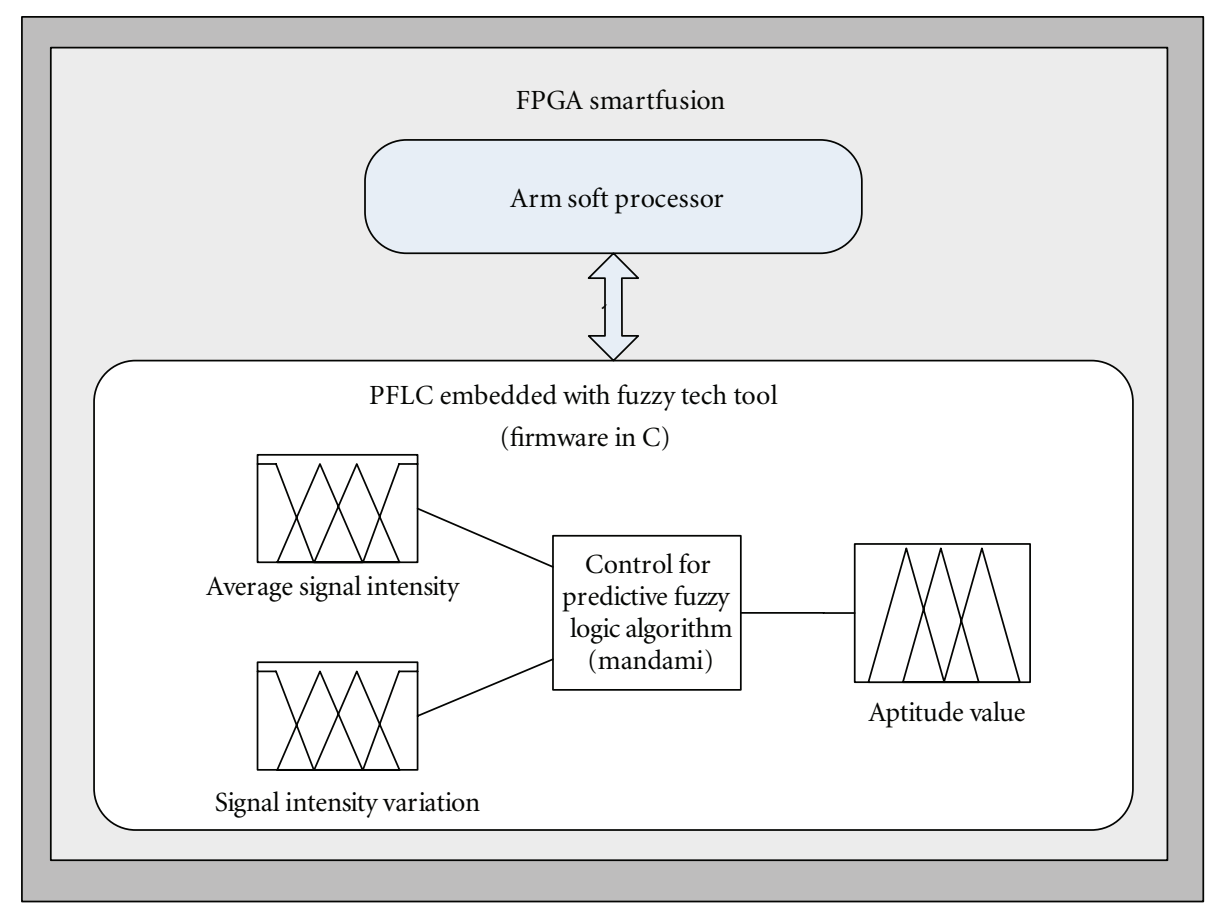

FIGURE 11: Fuzzy system embedded in FPGA smartfusion.

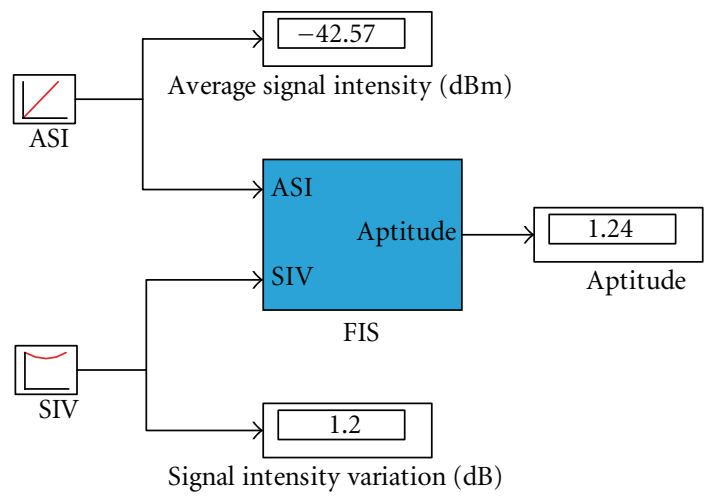

Figure 12: Aptitude value for the source AP.

TABLE 5: ASI values.

\begin{tabular}{lc}
\hline ASI $(\mathrm{dBm})$ & Time $(\mathrm{sec})$. \\
\hline-78.44 & 2 \\
-76.55 & 4 \\
-74.54 & 6 \\
-72.54 & 8 \\
$\ldots$ & $\ldots$ \\
-20 & $n$ \\
\hline
\end{tabular}

versus the source's, but it is until the MS gets to point 4, when the conditions are set to trigger a Handoff. Figure 14 illustrates the PFLC embedded for eleven channels, with the selection of a best aptitude.

Under normal conditions, IEEE 802.11 proceeds to a scanning process of eleven channels to determine the one

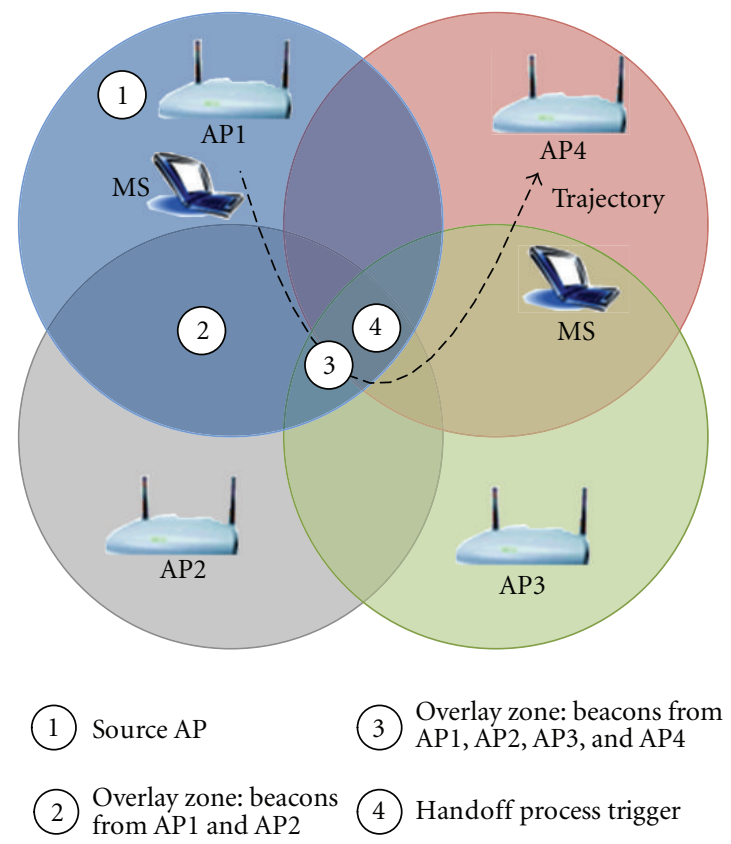

Figure 13: Multiple APs.

with the highest signal strength, and then decide which one will be the target AP. In our proposal, the scanning process is reduced to a single channel, since the MS knows in advance which AP has the higher aptitude.

Figure 15 shows the three-dimensional control surface computed by the Mamdani-type FLPC. The axes values correspond to the ASI, SIV, and Aptitude. As can be 


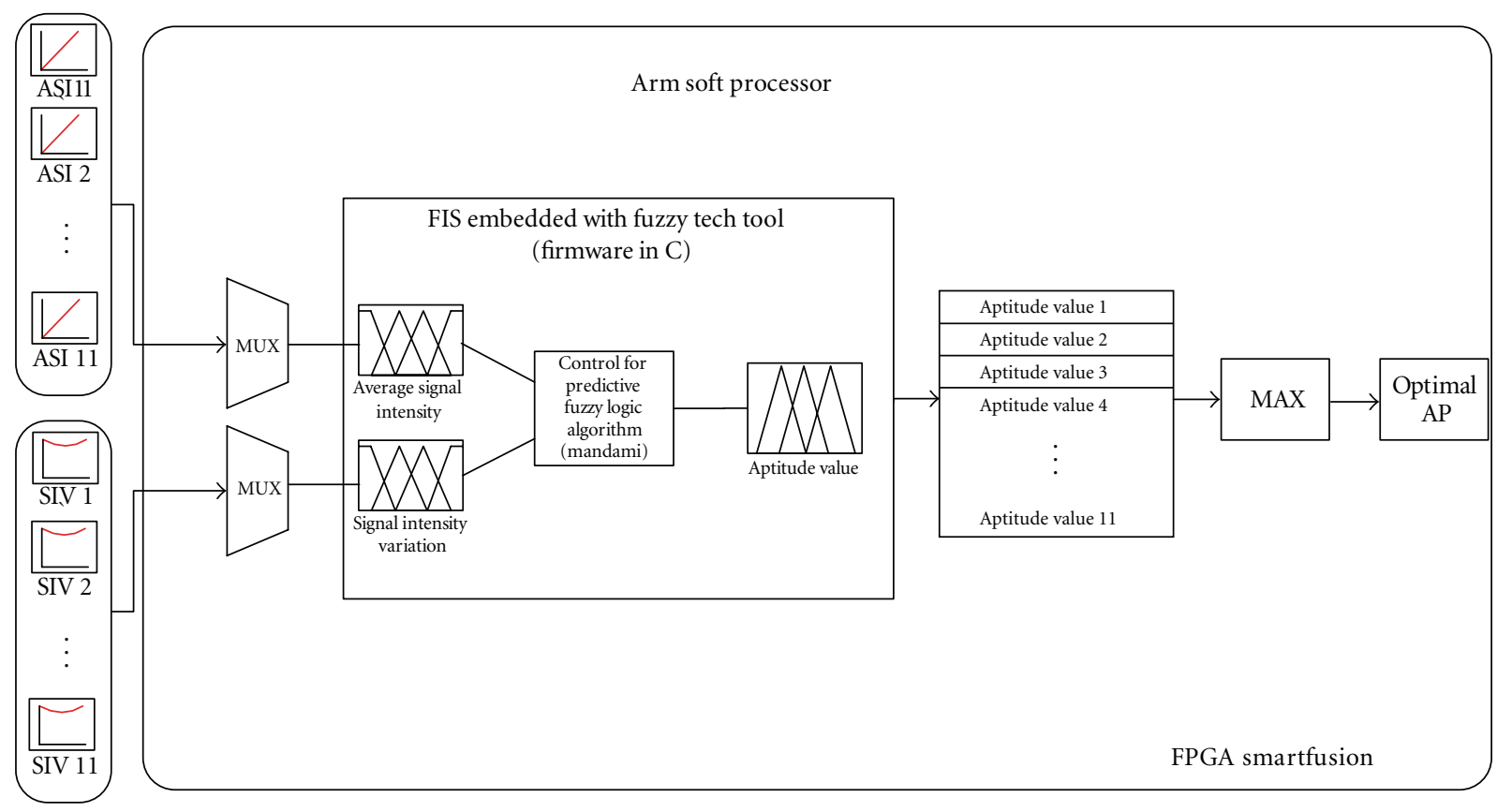

FIGURE 14: Fuzzy control in operation with multiple APs.

TABLE 6: Comparison of the output using the fuzzy toolbox of Matlab and the FLPC in the FPGA.

\begin{tabular}{lcccc}
\hline & \multicolumn{2}{c}{ Input } & \multicolumn{2}{c}{ Output } \\
& ASI & SIV & Matlab & FLPC in the FPGA \\
\hline 1 & -23.27 & 1.03 & 1.38 & 1.50 \\
2 & -54.22 & -1.34 & -0.85 & -0.76 \\
3 & -35.10 & 0.85 & 1.22 & 1.28 \\
4 & -42.77 & -2.86 & -0.16 & -0.14 \\
5 & -60.35 & -0.25 & -0.75 & -0.69 \\
6 & -71.97 & 0.36 & -0.31 & -0.32 \\
7 & -56.13 & 2.83 & 0.28 & 0.27 \\
8 & -44.30 & -2.68 & -0.22 & -021 \\
9 & -80.00 & -0.10 & -0.60 & -0.55 \\
10 & -20.21 & 0.73 & 1.35 & 1.50 \\
\hline
\end{tabular}

seen, when SIV is negative and ASI is minimum, aptitude values are negative, indicating an AP which should not be considered as the destination for a handoff. The highest values of Aptitude $(1<z \leq 2)$ are obtained from the conjunction of high ASI values $(-30<x \leq-20 \mathrm{dBm})$ and positive SIV values $(2<y \leq 3)$.

Figure 15 shows the control surface of the fuzzy handoff controller. This surface controls the rate of change of the AVS and SIV signals and it was obtained experimentally; however it can be changed using any method such as the simple tuning method [28], or considering other objectives, in this case it will be necessary to use a multiobjective optimization method in order to adapt the membership functions [29].

For a comparative analysis, in Table 6 we have the output for different values of ASI and SIV, of the PFLC designed with

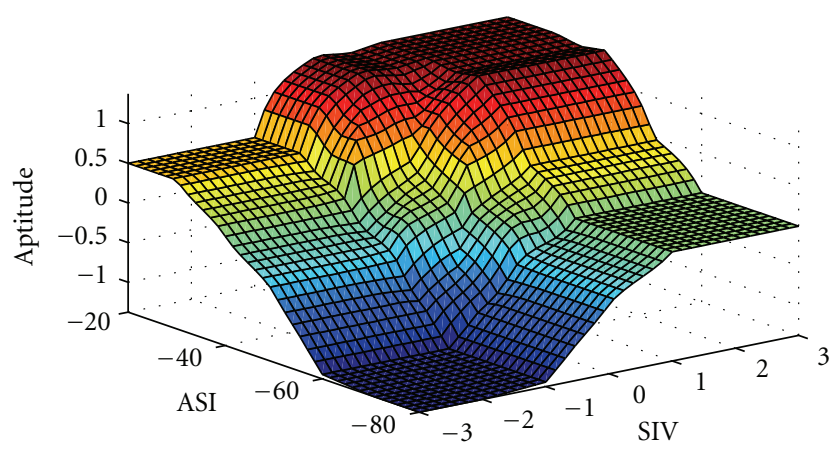

Figure 15: Fuzzy system control surface.

the fuzzy toolbox of Matlab [30], and the value obtained with our proposal. The differences are minimum.

\section{Conclusions}

VoIP is a real time application sensitive to time delays over $200 \mathrm{msec}$. Standard IEEE $802.11 \mathrm{can}$ have handoff delays of up to $300 \mathrm{msec}$. The capability to maintain uninterrupted VoIP connections while crossing through multiple WiFi cells is fundamental for this communication system's survival in the contested wireless arena. A predictive fuzzy logic control based on average signal intensity and signal intensity variation parameters as inputs to the fuzzy inference algorithm yields access point's aptitude value for up to eleven APs, selects the highest, and designates the best AP to perform a cell handoff. This approach reduces the handoff scanning time nearly $30 \mathrm{msec}$, and the complete $\mathrm{HO}$ to around $60 \mathrm{msec}$., thus providing better quality of service to 
VoIP and other real time applications, where multiple-cell arrangements are involved.

Soft computing tools (fuzzy logic, neural networks, and genetic algorithms) usage is on the rise to tackle highly complex problems, yielding for the most part satisfactory outcomes. Fuzzy logic has been widely used in control and prediction applications because of its human language parallelism, that makes it independent of rigorous analysis precision, thus providing analysis capabilities in uncertainty situations, where not-well-defined limits are found. This is the case in the decision-making process of the MS to launch a handoff while nearing its serving cell boundary. In a multiple-cell environment, received power and direction are computed in milliseconds intervals for the inference engine to decide the time and best fitted target AP.

Nowadays the Systems-on-Chip (SoC) are highly dense silicon chips, which integrate complete customized systems comprised of microprocessors and I/O devices to interface screens and keyboards, as is the case of cellular terminals. Therefore, the inclusion of a real-time high-performance fuzzy systems allows to achieve multiple tasks without sacrificing performance.

\section{Acknowledgment}

The authors would like to thank the Instituto Politécnico Nacional (IPN), Comisión de Operación y Fomento de Actividades Académicas (COFAA), and the Mexican Consejo Nacional de Ciencia y Tecnología (CONACYT) for supporting their research activities.

\section{References}

[1] Wi-Fi Alliance, Press Release, Austin, Tex, USA, 2012.

[2] Institute of Electrical and Electronics Engineers, "IEEE Standards Association, 802.11: Wireless LANs".

[3] LTE, WiMAX and WLAN Network Design, Optimization and Performance Analysis Leonhard Korowajczuk, John Wiley \& Sons, 2011.

[4] I. Purushothaman and S. Roy, "FastScan: A handoff scheme for voice over IEEE 802.11 WLANs," Wireless Networks, vol. 16, no. 7, pp. 2049-2063, 2010.

[5] ITU-TG.114, One-Way Transmission Time, 2003.

[6] Y. Maldonado, O. Montiel, R. Sepúlveda, and O. Castillo, "Design and simulation of the fuzzification stage through the Xilinx system generator," Studies in Computational Intelligence, vol. 154, pp. 297-305, 2008.

[7] J. A. Olivas, R. Sepúlveda, O. Montiel, and O. Castillo, "Methodology to test and validate a VHDL inference engine through the Xilinx system generator," Studies in Computational Intelligence, vol. 154, pp. 325-331, 2008.

[8] G. Lizárraga, R. Sepúlveda, O. Montiel, and O. Castillo, "Modeling and simulation of the defuzzification stage using Xilinx system generator and simulink," Studies in Computational Intelligence, vol. 154, pp. 333-343, 2008.

[9] S. Poorony, T. V. S. Urmila Priya, K. Udaya Kumara, and S. Renganarayanan, "FPGA based fuzzy logic controller for electric vehicle," Journal of the Institution of Engineers, vol. 45, no. 5, pp. 1-14, 2005.

[10] O. Montiel, R. Sepúlveda, Y. Maldonado, and O. Castillo, "Design and simulation of the type-2 fuzzification stage:
Using active membership functions," Studies in Computational Intelligence, vol. 257, pp. 273-293, 2009.

[11] R. Sepúlveda, O. Montiel, J. Olivas, and O. Castillo, "Methodology to test and validate a VHDL inference engine of a type-2 FIS, through the xilinx system generator," Studies in Computational Intelligence, vol. 257, pp. 295-308, 2009.

[12] R. Sepúlveda, O. Montiel, G. Lizárraga, and O. Castillo, "Modeling and simulation of the defuzzification stage of a type-2 fuzzy controller using the xilinx system generator and simulink," Studies in Computational Intelligence, vol. 257, pp. 309-325, 2009.

[13] R. Sepúlveda, O. Montiel, O. Castillo, and P. Melin, "Modelling and simulation of the defuzzification stage of a type-2 fuzzy controller using VHDL code," Control and Intelligent Systems, vol. 39, no. 1, pp. 33-40, 2011.

[14] R. Sepúlveda, O. Montiel, O. Castillo, and P. Melin, "Embedding a high speed interval type-2 fuzzy controller for a real plant into an FPGA," Applied Soft Computing Journal, vol. 12, no. 3, pp. 988-998, 2012.

[15] L. C. Godara, Handbook of Antennas in Wireless Communications, CRC Press, 2002.

[16] "Certified Wireless Network Administrator TM: Official Study Guide, Planet3 Wireless," 2002.

[17] A. R. Prasad and N. R. Prasad, 802.11 WLANs and IP Networking, Artech House, 2005.

[18] A. Mishra, M. Shin, Arbaugh, and W, "An empirical analysis of the IEEE 802.11MAC layer handoff process," ACM SIGCOMM Computer Communication Review, vol. 33, pp. 93-102, 2003.

[19] S. Shin, A. G. Forte, A. S. Rawat, and H. Schulzrinne, "Reducing MAC layer handoff latency in IEEE 802.11 wireless LANs," in Proceedings of the 2nd International Workshop on Mobility Management and Wireless Access Protocols (MobiWac '04), pp. 19-26, October 2004.

[20] C. S. Li, Y. C. Tseng, and H. C. Chao, "A neighbor caching mechanism for handoff in IEEE 802.11 wireless networks," in Proceedings of the International Conference on Multimedia and Ubiquitous Engineering (MUE '07), pp. 48-53, April 2007.

[21] C. Y. Chang, H. J. Wang, H. C. Chao, and J. H. Park, "IEEE 802.11 handoff latency improvement using Fuzzy Logic," Wireless Communications and Mobile Computing, vol. 8, no. 9, pp. 1201-1213, 2008.

[22] Y. Song, M. Liu, Z. Li, and Q. Li, "Handover latency of predictive FMIPv6 in IEEE 802.11 WLANs: a cross layer perspective," in Proceedings of the 18th International Conference on Computer Communications and Networks (ICCCN '09), Honolulu, Hawaii, USA, August 2009.

[23] E. H. Ong and J. Y. Khan, "QoS provisioning for VoIP over wireless local area networks," in Proceedings of the 11th IEEE Singapore International Conference on Communication Systems (ICCS '08), pp. 906-911, November 2008.

[24] C. G. Patil and M. T. Kolte, "An approach for optimization of handoff algorithm using fuzzy logic system," International Journal of Computer Science and Communication, vol. 2, pp. 113-118, 2011.

[25] A. M. Gharehbaghi and M. A. Badamchizadeh, "An improved method for fuzzy congestion control," International Journal of Computer and Network Security, vol. 2, no. 7, pp. 1-5, 2010.

[26] "Microsemi-Actel Corporation: SmartFusion Evaluation Kit User's Guide," USA, 2012.

[27] "Fuzzy tech: fuzzyTECH 5.5 user's manual, INFORM GmbH /Inform Software Corp," 2001.

[28] O. Montiel, R. Sepúlveda, P. Melin, O. Castillo, M. Á. Porta, and I. M. Meza, "Performance of a simple tuned fuzzy controller and a PID controller on a DC motor," in Proceedings 
of the IEEE Symposium on Foundations of Computational Intelligence (FOCI'07), pp. 531-537, April 2007.

[29] Y. Maldonado, O. Castillo, and P. Melin, "Optimization of membership functions for an incremental fuzzy PD control based on genetic algorithms," Studies in Computational Intelligence, vol. 318, pp. 195-211, 2010.

[30] R. Sepúlveda, O. Montiel-Ross, C. Juan Manzanarez, and E. Ernesto Quiroz, "Fuzzy logic predictive algorithm for wirelessLAN fast inter-cell handoff," Engineer Letters, vol. 20, no. 1, pp. 109-115, 2012. 

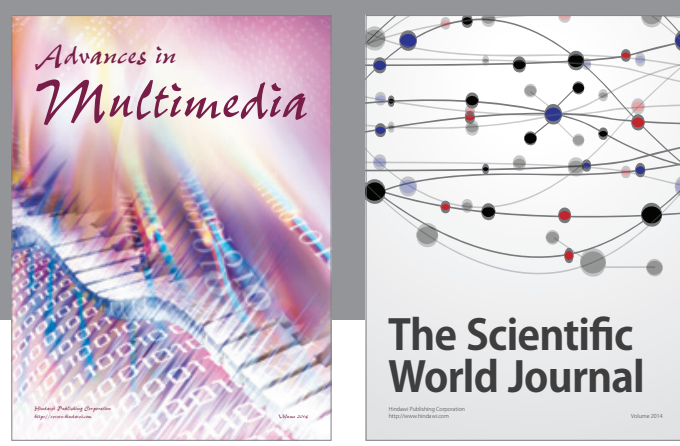

The Scientific World Journal
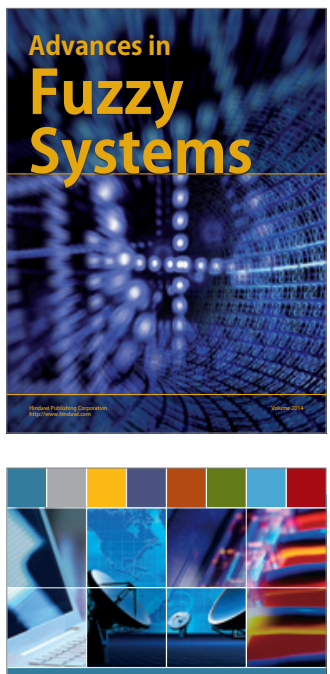

Computer Networks and Communications
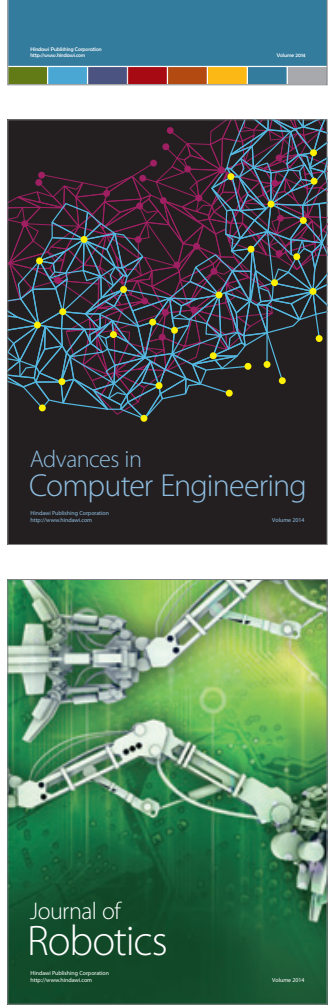
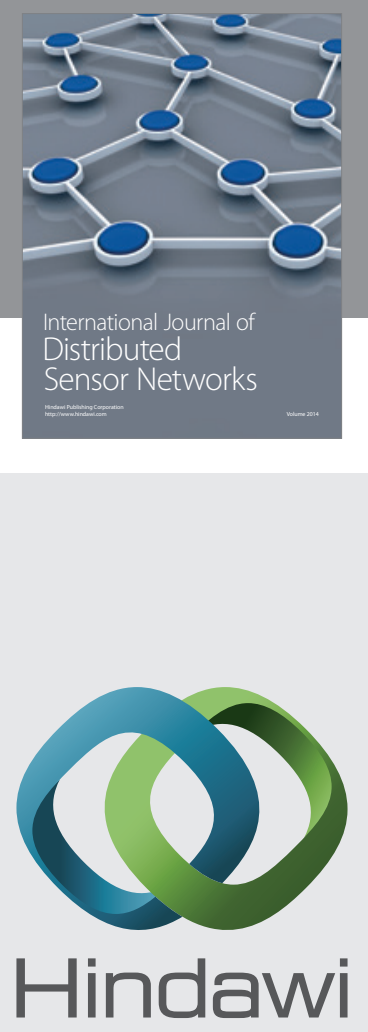

Submit your manuscripts at

http://www.hindawi.com
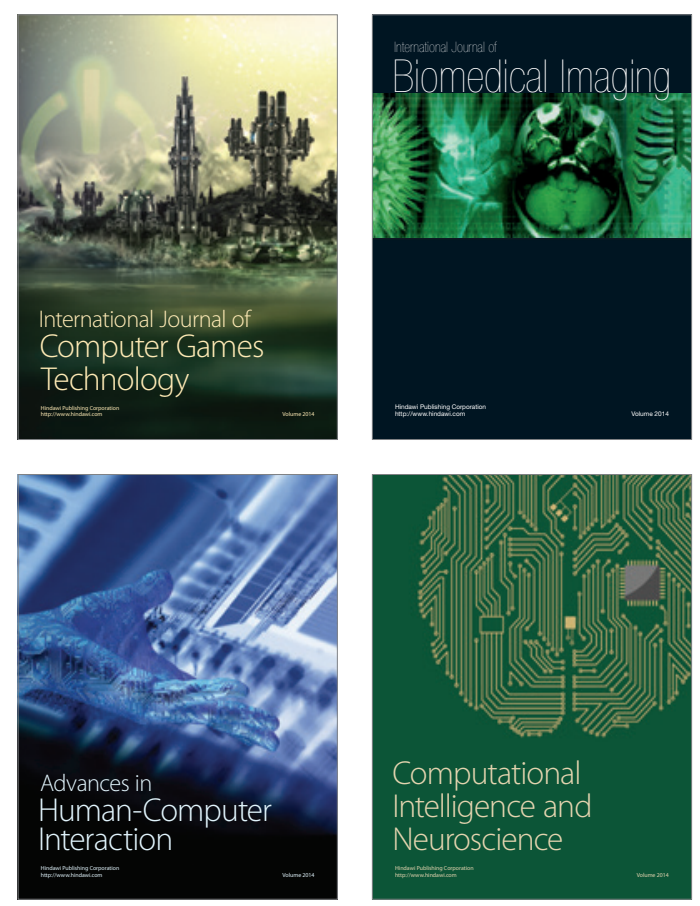
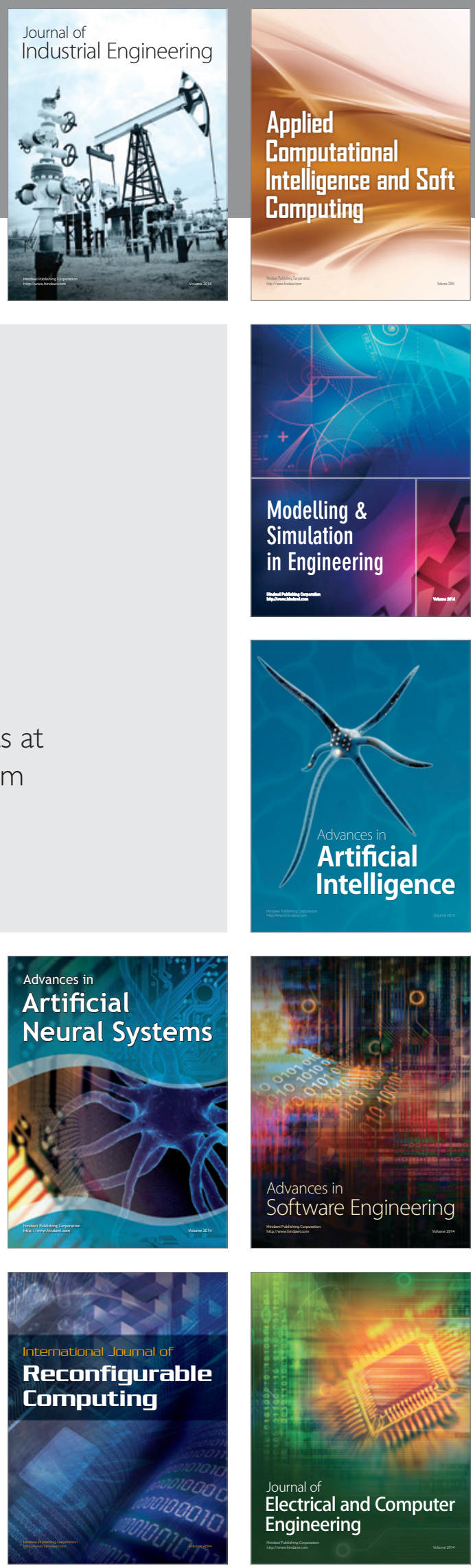